\title{
Blood Lactate or Lactate Clearance: Which Is Robust to Predict the Neurological Outcomes after Cardiac Arrest? A Systematic Review and Meta-Analysis
}

\author{
Bao-Chun Zhou, ${ }^{1}$ Zheng Zhang, ${ }^{2}$ Jian-Jun Zhu, ${ }^{1}$ Li-Jun Liu ${ }^{(D},{ }^{1}$ and Chun-Feng Liu ${ }^{3}{ }^{3}$ \\ ${ }^{1}$ Department of Emergency and Critical Care Medicine, The Second Affiliated Hospital of Soochow University, No. 1055, \\ San Xiang Road, Suzhou 215004, Jiangsu, China \\ ${ }^{2}$ Department of Nutrition and Food Hygiene, School of Public Health, Soochow University, 199 Renai Road, Suzhou, \\ Jiangsu Province 215123, China \\ ${ }^{3}$ Department of Neurology, The Second Affiliated Hospital of Soochow University, No. 1055, San Xiang Road, Suzhou 215004, \\ Jiangsu, China \\ Correspondence should be addressed to Chun-Feng Liu; feyicu@126.com
}

Received 26 July 2018; Revised 11 September 2018; Accepted 17 September 2018; Published 2 October 2018

Academic Editor: Ahmed Abdel-Latif

Copyright (C) 2018 Bao-Chun Zhou et al. This is an open access article distributed under the Creative Commons Attribution License, which permits unrestricted use, distribution, and reproduction in any medium, provided the original work is properly cited.

\begin{abstract}
Aims. Lactate and lactate clearance were supposed to be associated with cardiac arrest outcomes, but studies obtained different results. Thus, we conducted this meta-analysis to investigate the association between lactate or lactate clearance and neurological outcomes and their usefulness for prediction of neurological outcomes. Methods. We conducted a systematic search in PubMed, Web of science, EMBASE, Medline, and Google Scholar until May 1, 2018, for relevant studies. Studies reporting lactate, lactate clearance on admission, or other time points after admission associated with neurological outcomes were included in our analysis. Pooled effect date was shown as weighed mean difference (WMD) and 95\% confidence interval (CI). To measure the usefulness of lactate on admission to predict neurological outcomes, we also pooled the data of diagnostic test. Results. 23 studies involving 6720 cardiac arrest (CA) patients were included. Results from our analysis indicated that patients with good neurological outcomes tended to have a lower lactate level on admission (WMD: $-2.66 \mathrm{mmol} / \mathrm{L}, 95 \% \mathrm{CI}$ : -3.39 to -1.93 ) and $12 \mathrm{~h}, 24 \mathrm{~h}$, and $48 \mathrm{~h}$ after admission $(P<0.001)$. Furthermore, the pooled AUC for lactate level on admission to predict neurological outcomes was 0.77 (95\%CI: 0.73 0.80 ). However, a significant association between lactate clearance and neurological outcomes was only found in $24 \mathrm{~h}$ but not $12 \mathrm{~h}$ lactate clearance rate. Conclusions. Lactate levels on admission and all time points up to $48 \mathrm{~h}$ were associated with neurological outcomes after CA, whereas the association between lactate clearance and neurological outcomes was not so stable. Lactate was a more robust surrogate marker than lactate clearance to predict neurological outcomes after CA.
\end{abstract}

\section{Introduction}

Cardiac arrest (CA) presents a serious public health concern with high mortality cause by various pathogenesis such as anoxia, drug, electrolyte disturbance, low temperature, and hypoglycemia [1]. In Europe and north American, CA causes more than 600,000 cases per year [2], and the survival rate at hospital discharge is very low. Two major reasons for the mortality after resuscitation are postresuscitation circulatory failure and postanoxic neuro injury. Many researches have performed studies to develop tools for predicting the neurological outcomes. Biological markers such as neuron-specific enolase and S-100 $\beta$ protein have been demonstrated to be efficient predictors for neurological outcomes after CA. But these markers need longer time for detection. Therefore, a facility marker to predict neurological is needed in clinical practice. Lactate and lactated clearance are supposed to be that kind of marker.

Lactate is a product of pyruvate reduction during glycolysis. During CA, tissue hypoxia leads to accumulation of pyruvate and therewith the accumulation of lactate. The level of circulation lactate is likely to be a good marker of tissue hypoxia which is also recommended by Utstein guidelines. Not only lactated level but also a lactate clearance 
from serial determination of lactate is supposed to associated with cardiac arrest outcomes of CA $[3,4]$, and a serial determination of lactate is also recommended in CA survival after return of spontaneous circulation (ROSC) $[5,6]$. But studies obtain discrepant results [3, 4, 7-11]. This also leads to some confusions in the application of lactate or lactate clearance to predict neurological outcomes in clinic. In order to figure out the relationship between lactate or lactate clearance at different time points and neurological outcomes after CA and compare the efficiency of lactate or lactate clearance in predicting neurological outcomes after CA, we carry out a meta-analysis to summarize the current evidence.

\section{Materials and Methods}

2.1. Search Strategy. We carried out a literature search on PubMed database, Medline, EMBASE, Web of Science, and Google Scholar. We searched these databases until May 1, 2018, for studies containing the following keyword and/or Medical Subject Heading terms: (1) lactate OR hyperlactatemia OR lactate acid OR lactate clearance OR lactic clearance OR lactate normalization OR lactate kinetics, (2) heart arrest OR out of hospital cardiac arrest OR OHCA, and (3) neurological outcome OR cardiac arrest outcome OR CA outcome.

Besides, we also reviewed the reference lists of relevant articles to identify additional studies. Letters to author or abstract were scrutinized and excluded because they lacked sufficient data for analysis.

2.2. Study Selection. Studies were selected into our metaanalysis based on the following criteria: (1) carried on nontrauma cardiac arrest patients; (2) reported lactate level on admission or other time points after admission related to neurological outcomes; (3) reported lactate clearance related to neurological outcomes; (4) reported the detailed data of prognostic test. Studies were excluded in the following cases: (1) if it did not report lactate level related to neurological outcomes, (2) letter to author, and (3) study abstract.

2.3. Data Extraction. The following data were extracted from each eligible study: the first author's last name, year of publication, study design, geographic location, number of subjects, mean age of subjects, gender of subjects, percentage of initial shockable rhythm, location of CA, outcome followup time, therapeutic hypothermia, the sources where lactate measures from, neurological outcomes, and mean lactate level on admission. Besides, the data of serum lactate level and lactate clearance related to neurological outcomes at different time points were also extracted. To make an overview of lactate diagnostic efficiency, date of diagnostic test was also extracted. Lactate cut-off point, lactate measured time, neurological outcome measured time, and absolute value of true positive, true negative, false positive, and false negative were retrieved or developed $2 \times 2$ contingency table. Study selection and date extraction were conducted independently by two authors (Z-BC and Z-Z), with any disagreements resolved by consensus.
2.4. Definition of Neurological Outcome. The primary end point was neurological outcomes at discharge from hospital or another follow-up time. Most of studies used GlasgowPittsburgh cerebral performance categories (CPC) to measure the neurological outcome after CA [3, 10, 12-18, 2031]. Good neurological outcome was defined as a CPC of 1-2 and poor neurological outcome was defined as a CPC of 3-5. One study employed Glasgow Coma Score (GCS) on hospital discharge to measure the neurological outcome [19]. Good neurological outcome was defined as a GCS of 14-15. Another study adopting modified Rankin scale (mRS) to measure neurological outcome and good neurological outcome was defined as a mRS of 0-3.

2.5. Methodological Quality Assessment. The methodological quality assessment of eligible studies was appraised using Newcastle-Ottawa Scale (NOS) [32]. Articles with scores $<4$, between 4 and 6 , or $>6$ were considered as low, intermediate, or high quality, respectively. The assessment was also carried out by two authors (Z-BC and $\mathrm{Z}-\mathrm{Z}$ ). If there was any disagreement, a third author would reevaluate the original study.

2.6. Statistical Methods. Effect size of lactate difference between different neurological outcomes patients was defined as weighed mean difference (WMD) and 95\% confidence interval (CI). Articles reported data as interquartile range and median $[4,10,13-17,19,21,23-25,27,30,31]$. We converted data into mean and s.d. according to the method developed by Wan [33]. The unit of lactate was uniformed to $\mathrm{mmol} / \mathrm{L}$. Lactate measured at initial, on the arrival of hospital, emergency department, or ICU was defined as admission lactate level. Lactate measured within one hour after admission was also defined as lactate on admission. Besides, we also analyzed lactate level at $12 \mathrm{~h}, 24 \mathrm{~h}$, and $48 \mathrm{~h}$ after admission related to neurological outcomes. We used the Cochran $\mathrm{Q}$ and $\mathrm{I}^{2}$ statistics to assess heterogeneity among studies. For the $\mathrm{Q}$ statistic, a $P$ value $<0.1$ was considered statistically significant heterogeneity. For the $\mathrm{I}^{2}$ statistics, a value greater than $50 \%$ was considered high heterogeneity [34]. We used a random-effects model to estimate WMD in case of heterogeneity. Statistical synthesis and data analysis of diagnostic test were conducted according to the method introduced by Lee [35]. Sensitivity, specificity, likelihood ratios, diagnostic odds ratios, and receiver operating characteristic curves (SROC) were pooled using the DerSimonian and Laird method (random-effects model). To analyze the threshold effect, Spearman correlation coefficient of sensitivity and 1-specificity was calculated.

Considering that the differences in trails design and baseline characteristic of patients may contribute to the obvious heterogeneity observed in our analysis, we also designed subgroup analysis and metaregression analysis to explore the vital baseline factors. We carried out subgroup analysis according to mean age (adult versus old), CA location (OHCA versus OHCA/IHCA) (OHCA: out of hospital cardiac arrest; IHCA: in hospital cardiac arrest), initial shockable rhythm (high percentage versus low percentage), quality of included studies (high versus intermediate), data 


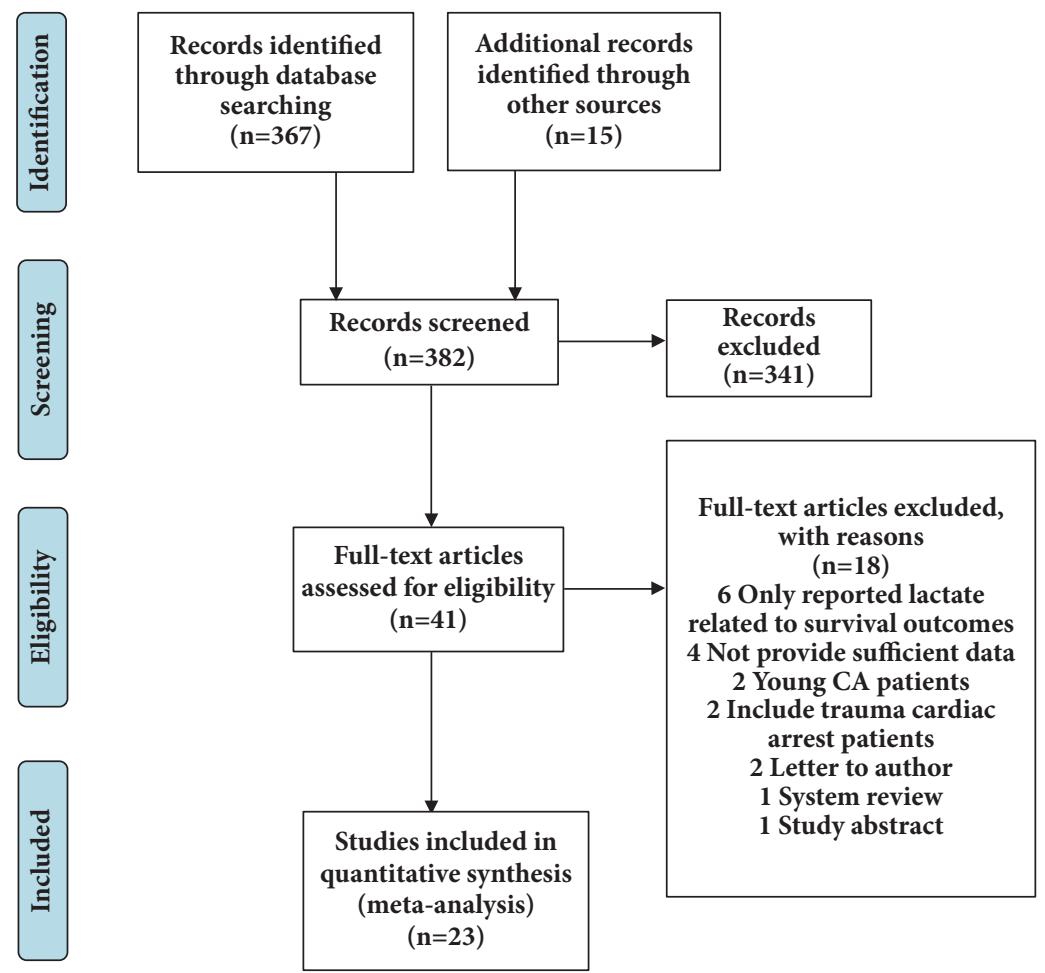

FIGURE 1: Flow diagram of study selection process.

transformation (Yes versus No), and outcome follow-up time (long versus short-term). Old people were defined as patients above 65 years old. Long-term follow-up was defined as measured neurological outcomes 3 months or longer after CA. Metaregression analysis carried out subgroup analysis according to mean age, percentage of initial shockable rhythm, and mean lactate level on admission.

Sensitivity analysis was employed to assess the stability of the meta-analysis. The publication bias was assessed using Egger [36] and Begg [37] tests, and $P$ value less than 0.05 was considered to have a significant publication bias. All statistical analyses were performed using the STATA version 14.0 and Meta-DiSc version 1.4. Except where otherwise specified, a $P$ value $<0.05$ was considered to be statistically significant.

\section{Results}

3.1. Literature Search. The systematic literature search yielded 382 potentially relevant records (Figure 1). After excluding duplicates and clearly irrelevant publications by reading titles and abstracts, we obtained 41 full articles of potentially relevant studies for a further evaluation. After full-text reviews, 18 out of 41 articles were excluded. Six articles reported lactate levels related to survival outcome; 4 articles did not provide sufficient data; 2 articles carried on CA patients under the age of 18; 2 articles included trauma cardiac arrest patients; 2 articles were letter to author; 1 was system review; and 1 was study abstract. Finally, 23 articles fulfilled our inclusion criteria $[3,4,10,12-31]$.
3.2. Study Characteristics. Baseline information of included studies was provided in Table 1 . The included 23 studies contained data from 6720 CA patients. 14 studies were retrospective design. Studies were conducted in countries including Japan $(n=7)$, Korea $(n=5)$, USA $(n=4)$, Australia $(n=2)$, France $(n=2)$, Belgium $(n=1)$, Finland $(n=1)$, and Norway $(n=1)$. The location of CA of patients includes OHCA $(n=19)$ and IHCA/OHCA $(n=4)$. The mean age of patients ranged from to 51 to 78 . The outcomes measuring time ranged from discharge from hospital (DC) to 6 months. Quality scores based on NOS scale ranged from 4 to 8.14 studies were defined as high quality (Table S1).

3.3. Lactate Level and Neurological Outcomes. 22 articles $[3,4,10,13-31]$ reported lactate levels at different time points related to neurological outcomes, which contained 6,553 participants. These studies were highly heterogeneous within $\mathrm{I}^{2}>90 \%$. Consequently, the random effect model was utilized to combine effect size. The WMD on admission was $-2.66 \mathrm{mmol} / \mathrm{L}$ (95\%CI: -3.39 to $-1.93, P<0.001, \mathrm{I}^{2}=91.1 \%$ ) (Figure 2). More importantly, a significant result was also found at other time points. The WMD for $12 \mathrm{~h}$ after admission was $-1.32 \mathrm{mmol} / \mathrm{L}$ (95\%CI: -1.82 to $-0.82, P<0.001, \mathrm{I}^{2}=87.4$ ). The WMD for $24 \mathrm{~h}$ after admission was $-0.85 \mathrm{mmol} / \mathrm{L}$ (95\%CI: -1.45 to $\left.-0.25, P=0.005, \mathrm{I}^{2}=96.4\right)$. The WMD for $48 \mathrm{~h}$ after admission was $-0.64 \mathrm{mmol} / \mathrm{L}$ (95\%CI: -1.17 to $-0.12 P=0.016$, $\mathrm{I}^{2}=98.1$ ) (Figure 3).

In addition, we also pooled date of diagnostic test to evaluate the diagnostic efficiency of lactate level on admission. 


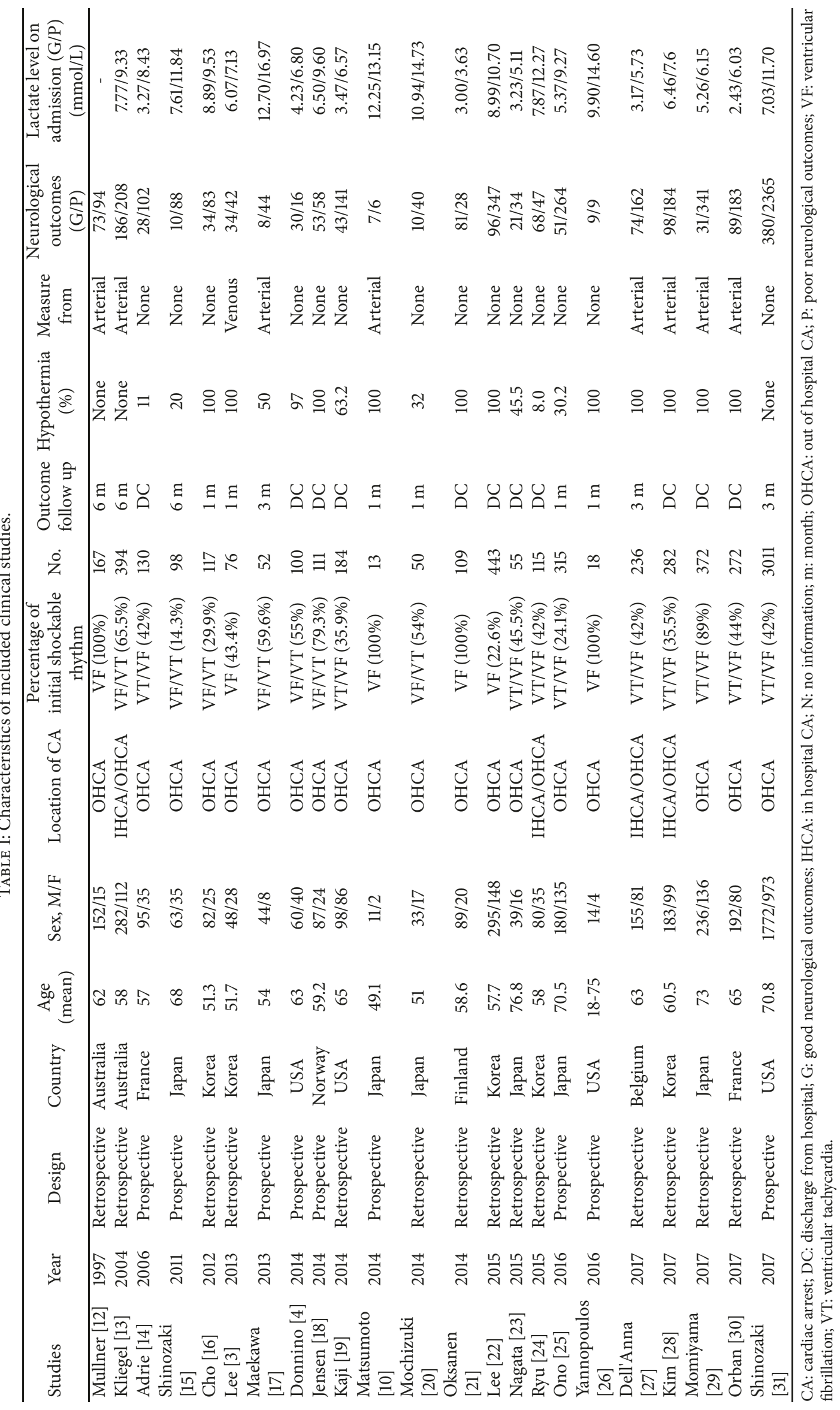




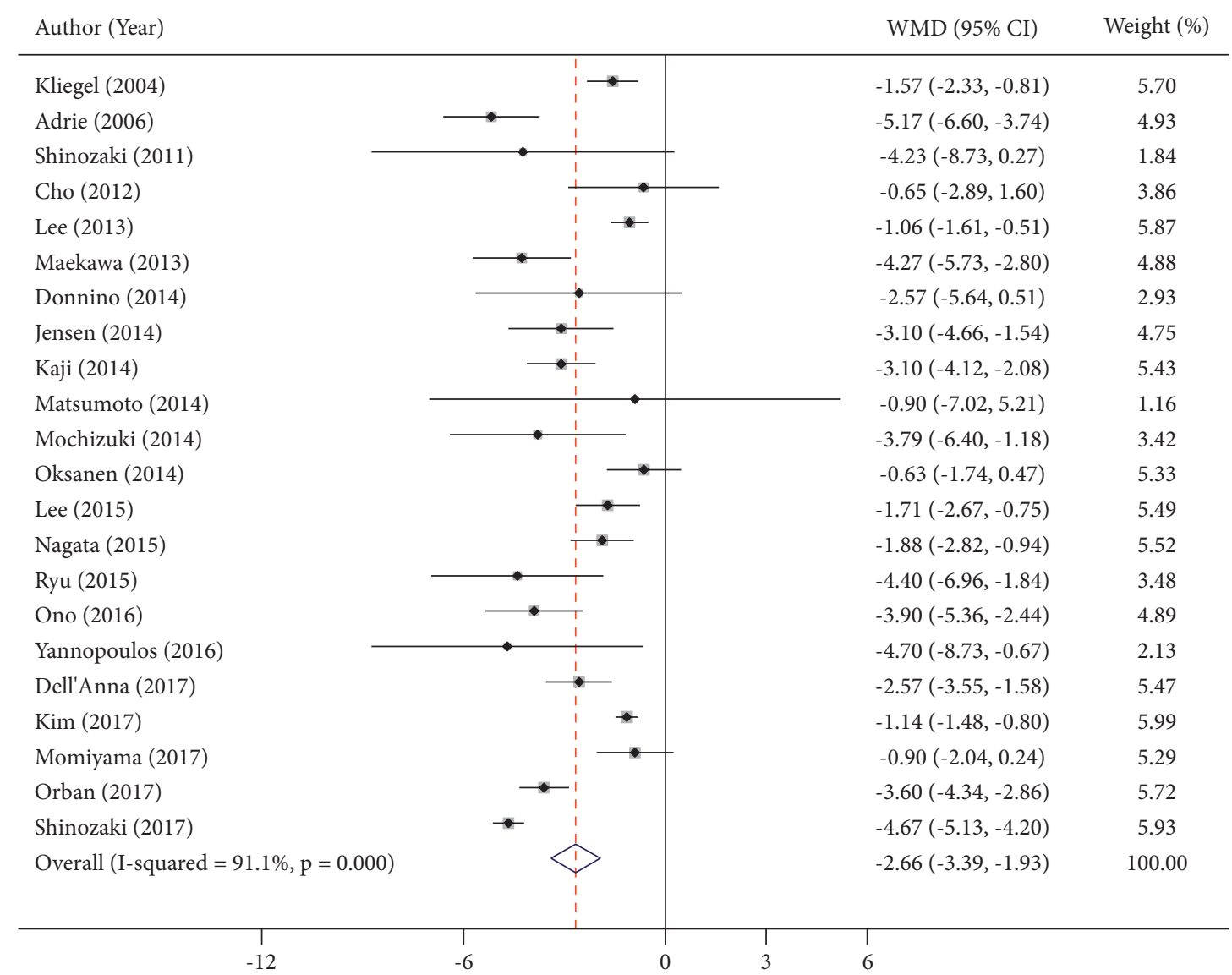

FIgURE 2: Summary weighed mean difference (WMD) and 95\% confidence intervals for lactate on admission between good and poor neurological outcomes $(\mathrm{mmol} / \mathrm{L})$.

Nine studies reported the date of predict diagnostic test [12, $15,17,22,23,25,27,29,31]$ and the information was shown in Table S2. But only six studies reported sufficient data to calculate the number of true positive (TP), false positive (FP), false negative $(\mathrm{FN})$, and true negative (TN) $[12,15,17,23,29,31]$. The Spearman correlation coefficient was $0.771(P=0.072)$, suggesting that there was no threshold effect. The pooled sensitivity was 69\% (95\%CI: 64\%-73\%); specificity was $77 \%$ (95\%CI: 75\%-79\%); positive likelihood ratio was 2.98 (95\%CI: 2.69-3.29); negative likelihood ratio was 0.41 (95\%CI: $0.35-$ 0.48 ); and diagnostic odds ratios (DOR) was 5.90 (95\%CI: 3.92-8.89). Receiver operating characteristic curves (SROC) did not show a curve in the top left corner of the plot which further indicated the lack of threshold effect and AUC of SROC curve was 0.77 (95\%CI: 0.73-0.80) which indicated a moderate diagnostic efficiency of lactate level on admission to predict neurological outcomes after CA. (Figure 4).

Due to limited studies in other time points, the subgroup analysis was only conducted on admission. Subgroup analysis was conducted based on mean age of CA patients, CA location outcome follow-up time, and study design. Age of patients, CA location or outcomes follow-up time did not substantially invert the difference of admission lactate level between good, and poor neurological outcomes or change the heterogeneity between studies (Table 2), whereas when the studies were stratified by study design, heterogeneity was vanished in studies with a prospective design (WMD: $-4.47 \mathrm{mmol} / \mathrm{L}, 95 \% \mathrm{CI}:-4.86$ to $\left.-4.08, P=0.432, \mathrm{I}^{2}=0.2 \%\right)$. In addition, the results of metaregression analysis showed that lactate level on admission would be another important heterogeneity source $(P=0.049<0.05)$ (Figure. S1).

3.4. Lactate Clearance and Neurological Outcome. Four studies measured lactate clearance related to neurological outcomes $[3,4,10,28]$. Three studies reported $12 \mathrm{~h}$ and $24 \mathrm{~h}$ lactate clearance of patients with different neurological outcomes $[3,4,28]$ and the remaining study only reported $24 \mathrm{~h}$ lactate clearance of patients between good and poor neurological outcomes patients [10]. A significant higher 24h lactate clearance was found in patients with good neurological outcomes (WMD: $8.32 \%, 95 \% \mathrm{CI}$ : 6.61 to $10.02, P<0.001$, $\mathrm{I}^{2}=0.0 \%$ ), but there was no significant difference of $12 \mathrm{~h}$ lactate clearance between different neurological outcomes (WMD: $13.70 \%$, 95\%CI: -4.18 to $31.57, P<0.001, \mathrm{I}^{2}=98.1 \%$ ) (Figure 5).

3.5. Sensitivity and Publication Bias Analysis. In sensitivity analysis, there was not one study substantiality that subverted the WMD of lactate between different neurological outcomes except lactate level at $48 \mathrm{~h}$ (Figure S2). The funnel plots 


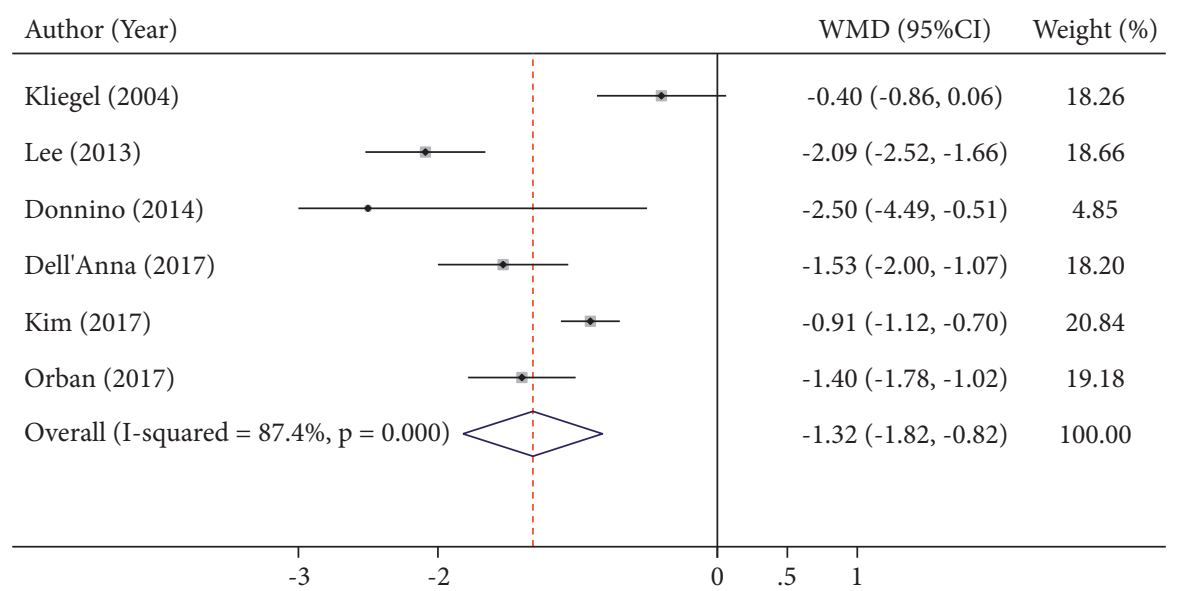

(a)

\begin{tabular}{|c|c|c|c|}
\hline Author (Year) & & WMD (95\%CI) & Weight (\%) \\
\hline Kliegel (2004) & & $-0.03(-0.28,0.22)$ & 16.39 \\
\hline Lee (2013) & & $-1.69(-2.01,-1.37)$ & 16.12 \\
\hline Donnino (2014) & & $-2.30(-3.87,-0.73)$ & 7.81 \\
\hline Matsumoto (2014) & & $-0.53(-1.72,0.67)$ & 10.08 \\
\hline Dell'Anna (2017) & & $-0.77(-1.02,-0.52)$ & 16.40 \\
\hline Kim (2017) & $\rightarrow$ & $-1.32(-1.51,-1.13)$ & 16.59 \\
\hline Orban (2017) & & $0.04(-0.15,0.23)$ & 16.60 \\
\hline Overall $(\mathrm{I}$-squared $=96.4 \%, \mathrm{p}=0.000)$ & & $-0.85(-1.45,-0.25)$ & 100.00 \\
\hline
\end{tabular}

(b)

\begin{tabular}{|c|c|c|c|}
\hline Author (Year) & & WMD (95\%CI) & Weight (\%) \\
\hline Kliegel (2004) & $\rightarrow$ & $-0.20(-0.32,-0.08)$ & 25.28 \\
\hline Lee (2013) & $\rightarrow$ & $-0.64(-0.82,-0.46)$ & 24.87 \\
\hline Dell'Anna (2017) & & $-0.53(-0.76,-0.31)$ & 24.48 \\
\hline Kim (2017) & $\rightarrow$ & $-1.20(-1.30,-1.10)$ & 25.36 \\
\hline Overall $(\mathrm{I}$-squared $=98.1 \%, \mathrm{p}=0.000)$ & & $-0.64(-1.17,-0.12)$ & 100.00 \\
\hline
\end{tabular}

(c)

Figure 3: Summary weighed mean difference (WMD) and 95\% confidence intervals for lactate at 12h (a), 24h (b), and 48h (c) between good and poor neurological outcomes $(\mathrm{mmol} / \mathrm{L})$.

showed no obvious dissymmetry in the shape of funnel plots in both time points (Figure S3). The $P$ value of Begg rank correlation test and Egger liner regression test for lactate on admission was 0.463 and 0.346 , respectively. In other time points, the results of Begg and Egger test also suggested no evidence of publication bias $(P>0.05)$.

\section{Discussion}

To the best of our knowledge, this was the first meta-analysis paying a close attention to the association and prognostic value of blood lactate and lactate clearance on CA neurological outcomes through systematically reviewing 23 relevant 

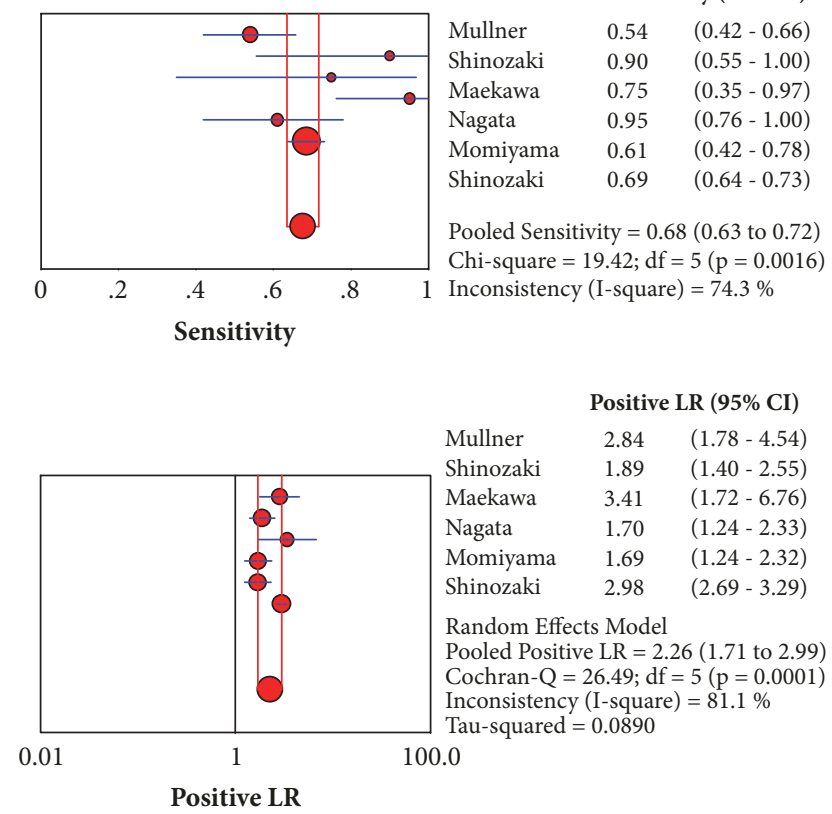

\section{Sensitivity (95\% CI)}

$0.54 \quad(0.42-0.66)$

$0.90 \quad(0.55-1.00)$

$0.75 \quad(0.35-0.97)$

$0.95 \quad(0.76-1.00)$

$0.61-(0.42-0.78)$

$0.69 \quad(0.64-0.73)$

Pooled Sensitivity $=0.68$ (0.63 to 0.72$)$ Chi-square $=19.42 ; \mathrm{df}=5(\mathrm{p}=0.0016)$

Inconsistency (I-square) $=74.3 \%$

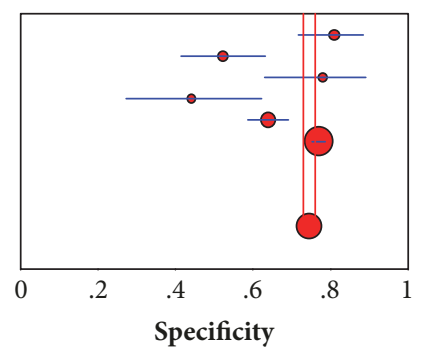

Mullner

Shinozaki

Maekawa

Nagata

Momiyama

Shinozaki

Pooled Specificity $=0.75(0.73$ to 0.76$)$

Chi-square $=62.95 ; \mathrm{df}=5(\mathrm{p}=0.0000)$

Inconsistency (I-square) $=92.1 \%$
Specificity (95\% CI)

$0.81 \quad(0.72-0.88)$

$(0.41-0.63)$

$0.78 \quad(0.63-0.89)$

$0.44 \quad(0.27-0.62)$

$0.64 \quad(0.59-0.69)$

$0.77 \quad(0.75-0.79)$
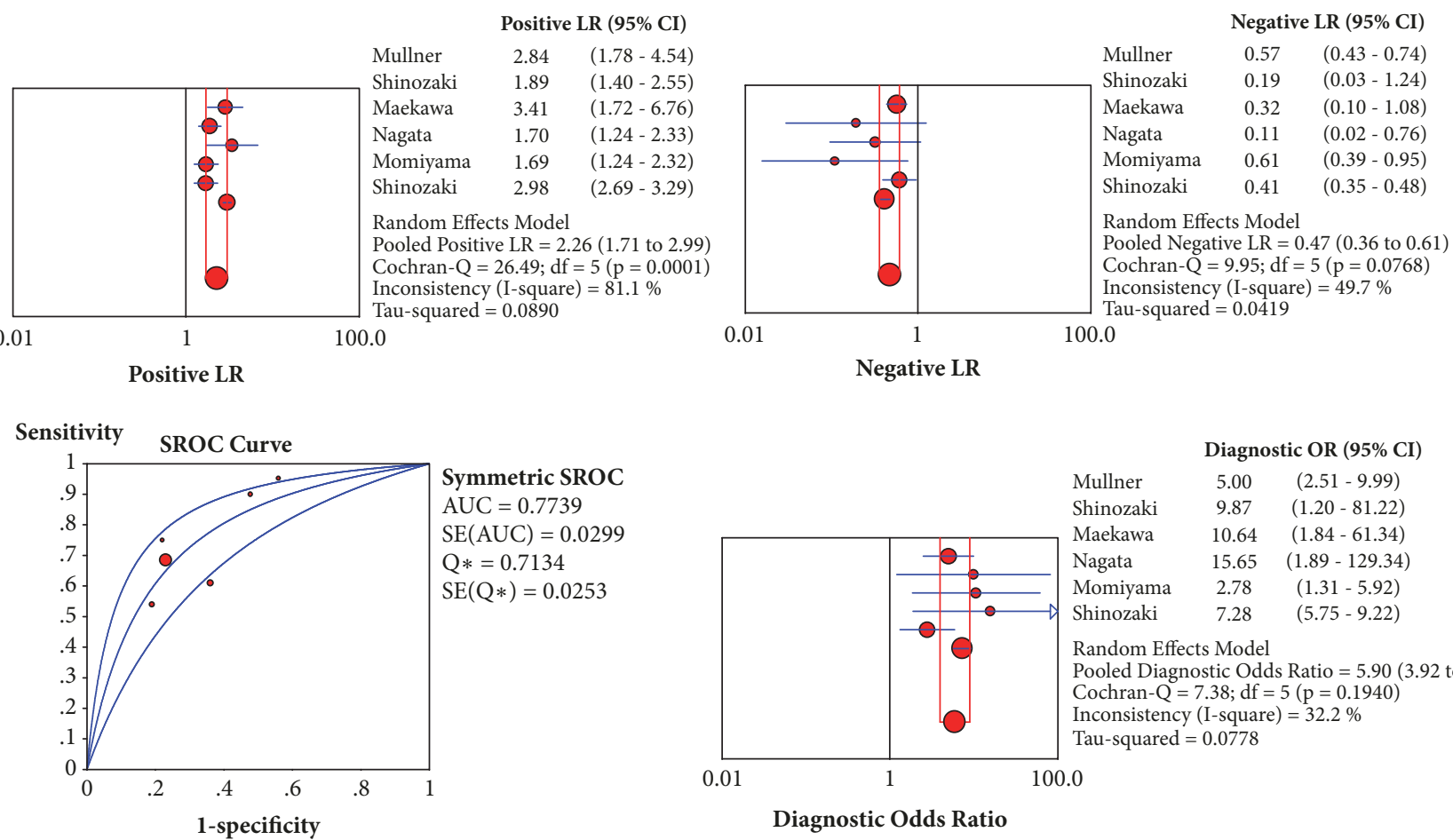

Symmetric SROC AUC $=0.7739$ $\mathrm{SE}(\mathrm{AUC})=0.0299$ $\mathrm{Q} *=0.7134$ $\mathrm{SE}(\mathrm{Q} *)=0.0253$

FIgURE 4: Pooled sensitivity, specificity, positive likelihood ratio (PLR), negative likelihood ratio (NLR), receiver operating characteristic curves (SROC), and diagnostic OR of serum lactate on admission for differentiating neurological outcomes after cardiac arrest.

studies. We found a significant relationship between lactate levels on admission and $12 \mathrm{~h}, 24 \mathrm{~h}$, and $24 \mathrm{~h}$ after admission were associated with neurological outcomes. Particularly lactate level on admission has the diagnostic ability to predict neurological outcomes after CA. But a significant association between lactate clearance and neurological outcomes was only found in $24 \mathrm{~h}$ but not $12 \mathrm{~h}$.

As an organ dysfunction metabolite, lactate level is related to prehospital care factors such as quality of bystander CPR, the duration of down time [38], and initial rhythm and post-CA care factors such as hypothermia therapy. Therefore lactate was measured routinely in cardiac arrest patients or even other critically ill patients to assess short or long-term prognosis [39]. In cardiac arrest patients, patients with a lower lactate level on admission have higher possibility of ROSC [40]. One recent meta-analysis by Debaty et al. [41] also revealed that patients with a lower serum lactate level on hospital admission were associated with better survival outcomes. In our case, we further explored the association between lactate or lactated clearance and neurological outcomes. And we successfully demonstrated the associations between lactate levels on admission and $12 \mathrm{~h}, 24 \mathrm{~h}$, and $48 \mathrm{~h}$ after admission and CA neurological outcomes. Additionally, we also found a robust prognostic value of lactate level on admission to predict neurological outcomes. But if we wanted to apply it as gold standard in post-CA care, it was necessary to combine lactate level with other markers (e.g., blood ammonia, vasopressor) [15, 42].

Studies reported that serum lactate level dramatically decreased within early time after admission (0-6h) but then slowly decreased $[3,4,28]$. A prospective designed multicenter study with 543 OHCA patients also documented that, despite the initial lactate level, effective lactate reduction over the first $6 \mathrm{~h}$ of postcardiac arrest care was associated with survival and good neurologic outcomes [43]. It was the early lactate clearance which dominated the decrease of serum lactate. In our study, there was no significant difference in $12 \mathrm{~h}$ lactate clearance rate between good and poor 


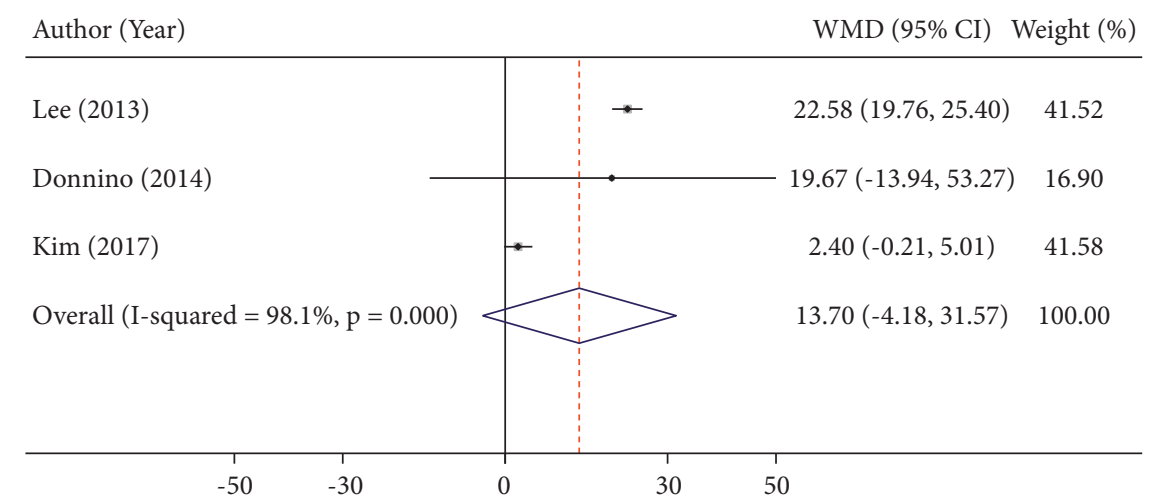

(a)

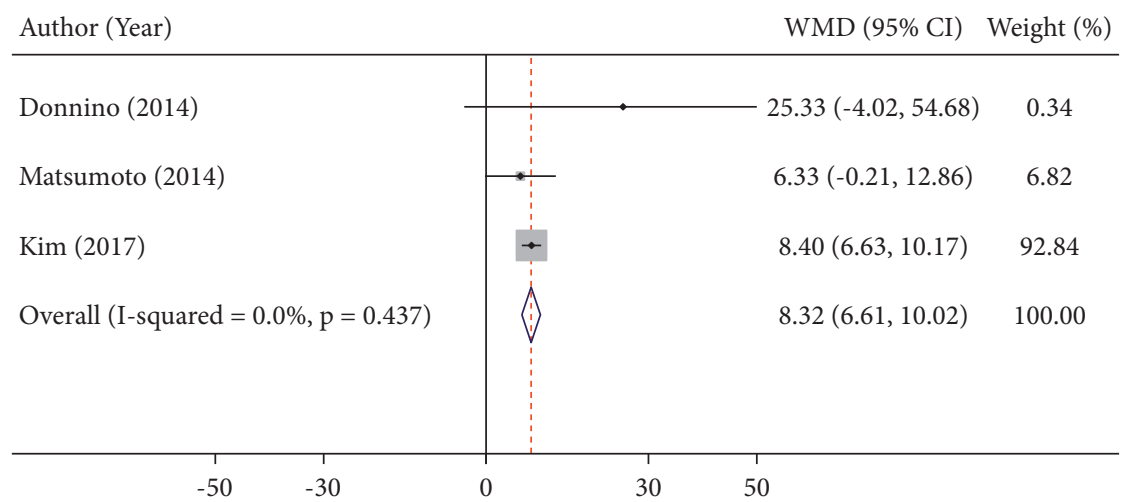

(b)

Figure 5: Summary weighed mean difference (WMD) and 95\% confidence intervals for lactate clearance rate at $12 \mathrm{~h}$ (a) and $24 \mathrm{~h}$ (b) after admission between good and poor neurological outcomes.

TABLE 2: Subgroup analysis of lactate on admission and neurological outcomes.

\begin{tabular}{|c|c|c|c|c|c|}
\hline Subgroups & No. of study & WMD & $95 \% \mathrm{CI}$ & $P$ for heterogeneity & $I^{2}(\%)$ \\
\hline \multicolumn{6}{|l|}{ Age } \\
\hline Adult & 14 & -2.27 & $-2.94,-1.69$ & 0.000 & 80.2 \\
\hline Old people & 8 & -3.17 & $-4.26,-1.60$ & 0.000 & 87.5 \\
\hline \multicolumn{6}{|l|}{ CA Location } \\
\hline OHCA & 18 & -2.79 & $-3.65,-1.93$ & 0.000 & 89.5 \\
\hline OHCA/IHCA & 4 & -1.93 & $-2.82,-1.04$ & 0.004 & 77.1 \\
\hline \multicolumn{6}{|l|}{ Outcome follow up time } \\
\hline Short-term $(<1 \mathrm{~m})$ & 18 & -2.52 & $-3.20,-1.84$ & 0.000 & 84.5 \\
\hline Long-term $(\geq 3 \mathrm{~m})$ & 4 & -3.10 & $-5.04,-1.16$ & 0.000 & 94.2 \\
\hline \multicolumn{6}{|l|}{ Study quality } \\
\hline Intermediate $(\leq 6)$ & 8 & -2.88 & $-4.12,-1.64$ & 0.000 & 77.2 \\
\hline $\operatorname{High}(>6)$ & 14 & -2.56 & $-3.48,-1.64$ & 0.000 & 93.6 \\
\hline \multicolumn{6}{|l|}{ Data transformation } \\
\hline Yes & 15 & -3.00 & $-3.84,-2.16$ & 0.000 & 86.4 \\
\hline No & 7 & -1.53 & $-2.09,-0.97$ & 0.03 & 56.9 \\
\hline \multicolumn{6}{|l|}{ Initial shockable rhythm } \\
\hline Low percentage $(<50 \%)$ & 13 & -2.83 & $-3.80,-1.87$ & 0.000 & 94.2 \\
\hline High percentage $(\geq 50 \%)$ & 9 & -2.28 & $-3.28,-1.29$ & 0.001 & 68.1 \\
\hline \multicolumn{6}{|l|}{ Study design } \\
\hline Retrospective & 13 & -1.92 & $-2.50,-1.35$ & 0.000 & 81.2 \\
\hline Prospective & 9 & -4.47 & $-4.86,-4.08$ & 0.432 & 0.2 \\
\hline
\end{tabular}


neurological outcomes patients. This was just in accord with the property of lactate metabolism kinetics. On the other hand, even we obtained a positive result in the association between $24 \mathrm{~h}$ lactate clearance and neurological outcomes, but 2 out of 3 included studies reporting negative results. The remaining one study reported that the association between lactate clearance and neurological outcomes disappeared at $12 \mathrm{~h}$ and $24 \mathrm{~h}$ after adjusting for potential confounders [28]. In summary, lactate clearance was not robust to predict neurological outcomes after CA.

Unique to recent meta-analysis which focuses on the lactate level on hospital admission and CA survival and neurological outcomes [41], we included more studies into our analysis, so the results were comprehensive and reliable. Another strength was that we conducted a summary of prognostic test to evaluate the neurological outcomes prognostic efficiency of lactate level on admission. Finally, we also explored the association between lactate levels after admission and lactate clearance related to neurological outcomes. This was exactly another unique advantage.

However, there still existed some limitations in our studies. Firstly, the heterogeneity between studies was obviously due to the huge discrepancy existing between studies, which also created some instability in our results to some extent. But these heterogeneities largely attributed to studies design as we found in our subgroup analysis. The bias of retrospective design in studies may contribute to the obvious heterogeneity in the analysis of lactate on admission and neurological outcomes. In addition to the study design, another important heterogeneity source we found in metaregression was the lactate level on admission. These two heterogeneity sources may contribute to not only the obvious heterogeneity existing in the analysis of lactate level on admission and neurological outcomes but also other analyses.

Secondly, even though we defined the association between lactate level on admission and CA neurological outcomes on OHCA and IHCA/OHCA, we did not confirm it in IHCA patients. Dell'Anna et al. found that there was no significant difference of lactate level between IHCA and OHCA [27]. Another system review obtained the result that lactate level on admission was associated with CA outcomes in IHCA patients treated with extracorporeal cardiopulmonary resuscitation [44], even though there was still huge heterogeneity between IHCA and OHCA population. Thirdly, the definition of lactate measuring time points was differed between studies. It was hard to make a uniform definition of lactate measured time points. Thus, it would be more reasonable to interpret with the time points in our study as a rough time division. Finally, the number of studies was limited in the analysis of lactate clearance and neurological outcomes which would also influence the stability of the results.

In conclusion, this meta-analysis took an overview of the relationship of lactate level and lactate clearance and CA neurological outcomes. We found that lactate level on admission and $12 \mathrm{~h}, 24 \mathrm{~h}$, and $48 \mathrm{~h}$ after admission was significantly associated with neurological outcomes. Additionally, lactate level on admission had a moderate neurological outcomes prognostic value. But, the lactate clearance was not robust to predict neurological outcomes after CA. Lactate level was more robust than lactate clearance to predict neurological outcomes after CA. But in view of the heterogeneity between studies, these findings should be interpreted with cautions and still need more large population, prospective design, and clinical studies with superior quality control to verify.

\section{Data Availability}

All original data used to support the findings of this study are available from the corresponding author upon request.

\section{Conflicts of Interest}

The authors declared they have no conflicts of interest.

\section{Authors' Contributions}

Bao Chun Zhou was responsible for design and implementation of the study, as well as analysis and drafted the manuscript. Zheng Zhang partially performed data acquisition. Jian-Jun Zhu significantly contributed to the conception of the study. Chun-Feng Liu critically revised the manuscript.

\section{Acknowledgments}

The present study was supported in part by grants from Suzhou Key Medicine Project Fund of China (no. Szxk201504), Peking Union Medical Foundation-Ruiyi Emergency Medical Research Fund (no. R2017001), and Suzhou science and technology bureau Medicine Project Fund of China (no. SYS201506).

\section{Supplementary Materials}

Figure S1: metaregression plots of the association between mean changes in lactate concentrations after cardiac arrest and some baseline characteristics. Figure S2: sensitive analysis plots at different time points. Figure S3: funnel plots of included studies at different time points. Table S1: methodological quality assessment of included studies by NewcastleOttawa Scale. Table S2: the ability of lactate on admission to predict neurological outcomes. (Supplementary Materials)

\section{References}

[1] S. Ecc Committee and A. Task Forces of the American Heart, "2005 American Heart Association Guidelines for Cardiopulmonary Resuscitation and Emergency Cardiovascular Care”, Circulation, vol. 112, no.24 Suppl, pp. IV1-203, 2005.

[2] G. Nichol, E. Thomas, C. W. Callaway et al., "Regional variation in out-of-hospital cardiac arrest incidence and outcome," The Journal of the American Medical Association, vol. 300, no. 12, pp. 1423-1431, 2008.

[3] T. Lee, M. Kang, W. Cha et al., "Better lactate clearance associated with good neurologic outcome in survivors who treated with therapeutic hypothermia after out-of-hospital cardiac arrest," Critical Care, vol. 17, no. 5, p. R260, 2013.

[4] M. W. Donnino, L. W. Andersen, T. Giberson et al., "Initial lactate and lactate change in post-cardiac arrest: a multicenter validation study," Critical Care Medicine, vol. 42, no. 8, pp. 18041811, 2014. 
[5] J. P. Nolan, J. Soar, A. Cariou et al., "European Resuscitation Council and European Society of Intensive Care Medicine Guidelines for Post-resuscitation Care 2015. Section 5 of the European Resuscitation Council Guidelines for Resuscitation 2015.," Resuscitation, vol. 95, pp. 202-222, 2015.

[6] M. A. Peberdy, C. W. Callaway, R. W. Neumar et al., "Part 9: post-cardiac arrest care: 2010 American Heart Association Guidelines for Cardiopulmonary Resuscitation and Emergency Cardiovascular Care," Circulation, vol. 122, no. 3, pp. S768-S786, 2010.

[7] M. L. Gustafson, S. Hollosi, J. T. Chumbe, D. Samanta, A. Modak, and A. Bethea, "The effect of ethanol on lactate and base deficit as predictors of morbidity and mortality in trauma," The American Journal of Emergency Medicine, vol. 33, no. 5, pp. 607613, 2015.

[8] L.-P. Kamolz, H. Andel, W. Schramm, G. Meissl, D. N. Herndon, and M. Frey, "Lactate: Early predictor of morbidity and mortality in patients with severe burns," Burns, vol. 31, no. 8, pp. 986990, 2005.

[9] H. B. Nguyen, E. P. Rivers, B. P. Knoblich et al., "Early lactate clearance is associated with improved outcome in severe sepsis and septic shock," Critical Care Medicine, vol. 32, no. 8, pp. 1637$1642,2004$.

[10] H. Matsumoto, S.-I. Nihei, T. Endo et al., "Examination of relationship between lactate clearance and neurologic outcome in cardiac arrest induced by ventricular fibrillation," Journal of UOEH, vol. 36, no. 1, pp. 11-16, 2014.

[11] K. W. Kim, J. Lee, S. H. Choi, J. Huh, and S. H. Park, "Systematic review and meta-analysis of studies evaluating diagnostic test accuracy: A practical review for clinical researchers-part I. general guidance and tips," Korean Journal of Radiology, vol. 16, no. 6, pp. 1175-1187, 2015.

[12] M. Müllner, F. Sterz, H. Domanovits, W. Behringer, M. Binder, and A. N. Laggner, "The association between blood lactate concentration on admission, duration of cardiac arrest, and functional neurological recovery in patients resuscitated from ventricular fibrillation," Intensive Care Medicine, vol. 23, no. 11, pp. 1138-1143, 1997.

[13] A. Kliegel, H. Losert, F. Sterz et al., "Serial lactate determinations for prediction of outcome after cardiac arrest," Medicine, vol. 83, no. 5, pp. 274-279, 2004.

[14] C. Adrie, A. Cariou, B. Mourvillier et al., "Erratum: Predicting survival with good neurological recovery at hospital admission after successful resuscitation of out-of-hospital cardiac arrest: The OHCA score (European Heart Journal (2006) 27, (28402845))," European Heart Journal, vol. 28, no. 6, p. 774, 2007.

[15] K. Shinozaki, S. Oda, T. Sadahiro et al., "Blood ammonia and lactate levels on hospital arrival as a predictive biomarker in patients with out-of-hospital cardiac arrest," Resuscitation, vol. 82, no. 4, pp. 404-409, 2011.

[16] Y. M. Cho, Y. S. Lim, H. J. Yang et al., "Blood ammonia is a predictive biomarker of neurologic outcome in cardiac arrest patients treated with therapeutic hypothermia," The American Journal of Emergency Medicine, vol. 30, no. 8, pp. 1395-1401, 2012.

[17] K. Maekawa, K. Tanno, M. Hase, K. Mori, and Y. Asai, "Extracorporeal cardiopulmonary resuscitation for patients with outof-hospital cardiac arrest of cardiac origin: A propensitymatched study and predictor analysis," Critical Care Medicine, vol. 41, no. 5, pp. 1186-1196, 2013.
[18] H. Stær-Jensen, K. Sunde, T. M. Olasveengen et al., "Bradycardia during therapeutic hypothermia is associated with good neurologic outcome in comatose survivors of out-of-hospital cardiac arrest," Critical Care Medicine, vol. 42, no. 11, pp. 2401-2408, 2014.

[19] A. H. Kaji, A. M. Hanif, N. Bosson, D. Ostermayer, and J. T. Niemann, "Predictors of neurologic outcome in patients resuscitated from out-of-hospital cardiac arrest using classification and regression tree analysis," American Journal of Cardiology, vol. 114, no. 7, pp. 1024-1028, 2014.

[20] K. Mochizuki, H. Imamura, T. Iwashita, and K. Okamoto, "Neurological outcomes after extracorporeal cardiopulmonary resuscitation in patients with out-of-hospital cardiac arrest: A retrospective observational study in a rural tertiary care center," Journal of Intensive Care, vol. 2, no. 1, 2014.

[21] T. Oksanen, M. Skrifvars, E. Wilkman, I. Tierala, V. Pettilä, and T. Varpula, "Postresuscitation hemodynamics during therapeutic hypothermia after out-of-hospital cardiac arrest with ventricular fibrillation: a retrospective study," Resuscitation, vol. 85, no. 8, pp. 1018-1024, 2014.

[22] D. H. Lee, I. S. Cho, S. H. Lee et al., "Correlation between initial serum levels of lactate after return of spontaneous circulation and survival and neurological outcomes in patients who undergo therapeutic hypothermia after cardiac arrest," Resuscitation, vol. 88, pp. 143-149, 2015.

[23] K. Nagata, J. Tsurukiri, K. Ueno, and S. Mishima, "Predictors of neurological outcome in the emergency department for elderly patients following out-ofhospital restoration of spontaneous circulation," Signa Vitae, vol. 10, no. 1, pp. 53-63, 2015.

[24] J. Ryu, Y. H. Cho, K. Sung et al., "Predictors of neurological outcomes after successful extracorporeal cardiopulmonary resuscitation," BMC Anesthesiology, vol. 15, no. 1, 2015.

[25] Y. Ono, M. Hayakawa, K. Maekawa et al., "Fibrin/fibrinogen degradation products (FDP) at hospital admission predict neurological outcomes in out-of-hospital cardiac arrest patients," Resuscitation, vol. 111, pp. 62-67, 2017.

[26] D. Yannopoulos, J. A. Bartos, C. Martin et al., "Minnesota Resuscitation Consortium's Advanced Perfusion and Reperfusion Cardiac Life Support Strategy for Out-of-Hospital Refractory Ventricular Fibrillation," Journal of the American Heart Association, vol. 5, no. 6, 2016.

[27] A. M. Dell'Anna, C. Sandroni, I. Lamanna et al., "Prognostic implications of blood lactate concentrations after cardiac arrest: a retrospective study," Annals of Intensive Care, vol. 7, no. 1, 2017.

[28] J. C. Kim, B. K. Lee, D. H. Lee et al., "Association between lactate clearance during post-resuscitation care and neurologic outcome in cardiac arrest survivors treated with targeted temperature management," Clinical and Experimental Emergency Medicine, vol. 4, no. 1, pp. 10-18, 2017.

[29] Y. Momiyama, W. Yamada, K. Miyata et al., "Prognostic values of blood $\mathrm{pH}$ and lactate levels in patients resuscitated from outof-hospital cardiac arrest," Acute Medicine \& Surgery, vol. 4, no. 1, pp. 25-30, 2017.

[30] J. Orban, M. Novain, F. Cattet et al., "Association of serum lactate with outcome after out-of-hospital cardiac arrest treated with therapeutic hypothermia," PLoS ONE, vol. 12, no. 3, p. e0173239, 2017.

[31] K. Shinozaki, "Initial blood ammonia level is a useful prognostication tool in out-of-hospital cardiac arrest — multicenter prospective study (SOS-KANTO 2012 study)," Circulation Journal, vol. 81, no. 12, pp. 1839-1845, 2017. 
[32] G. Wells, B. Shea, and D. OConnell, The Newcastle-Ottawa Scale (NOS) for assessing the quality of nonrandomized studies in meta-analyses. http://www.ohri.ca/programs/clinicalepidemiology/oxford.htm.

[33] X. Wan, W. Wang, J. Liu, and T. Tong, "Estimating the sample mean and standard deviation from the sample size, median, range and/or interquartile range," BMC Medical Research Methodology, vol. 14, article 135, 2014.

[34] J. P. T. Higgins, S. G. Thompson, J. J. Deeks, and D. G. Altman, "Measuring inconsistency in meta-analyses," British Medical Journal, vol. 327, no. 7414, pp. 557-560, 2003.

[35] J. Lee, K. W. Kim, S. H. Choi, J. Huh, and S. H. Park, "Systematic review and meta-analysis of studies evaluating diagnostic test accuracy: A practical review for clinical researchers-part II. Statistical methods of meta-analysis," Korean Journal of Radiology, vol. 16, no. 6, pp. 1188-1196, 2015.

[36] M. Egger, G. D. Smith, M. Schneider, and C. Minder, "Bias in meta-analysis detected by a simple, graphical test," British Medical Journal, vol. 315, pp. 629-634, 1997.

[37] C. B. Begg and M. Mazumdar, "Operating characteristics of a rank correlation test for publication bias," Biometrics, vol. 50, no. 4, pp. 1088-1101, 1994.

[38] D. L. Carden, G. B. Martin, R. M. Nowak, C. C. Foreback, and M. C. Tomlanovich, "Lactic acidosis as a predictor of downtime during cardiopulmonary arrest in dogs," The American Journal of Emergency Medicine, vol. 3, no. 2, pp. 120-124, 1985.

[39] M. H. Weil and A. A. Afifi, "Experimental and clinical studies on lactate and pyruvate as indicators of the severity of acute circulatory failure (shock)," Circulation, vol. 41, no. 6, pp. 9891001, 1970.

[40] H. Koami, Y. Sakamoto, R. Sakurai et al., “Thromboelastometric analysis of the risk factors for return of spontaneous circulation in adult patients with out-of-hospital cardiac arrest," PLoS ONE, vol. 12, no. 4, p. e0175257, 2017.

[41] G. Debaty, V. Babaz, M. Durand et al., "Prognostic factors for extracorporeal cardiopulmonary resuscitation recipients following out-of-hospital refractory cardiac arrest. A systematic review and meta-analysis," Resuscitation, vol. 112, pp. 1-10, 2017.

[42] M. N. Cocchi, J. Miller, S. Hunziker et al., "The association of lactate and vasopressor need for mortality prediction in survivors of cardiac arrest," Minerva Anestesiologica, vol. 77, no. 11, pp. 1063-1071, 2011.

[43] K. Hayashida, M. Suzuki, N. Yonemoto et al., "Early lactate clearance is associated with improved outcomes in patients with postcardiac arrest syndrome: A prospective, multicenter observational study (SOS-KANTO 2012 study)," Critical Care Medicine, vol. 45, no. 6, pp. e559-e566, 2017.

[44] S. D’Arrigo, S. Cacciola, M. Dennis et al., "Predictors of favourable outcome after in-hospital cardiac arrest treated with extracorporeal cardiopulmonary resuscitation: A systematic review and meta-analysis," Resuscitation, vol. 121, pp. 62-70, 2017. 


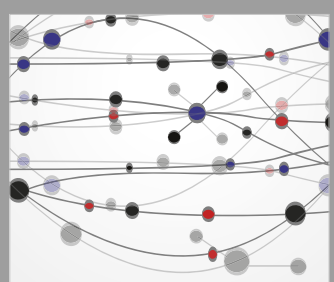

The Scientific World Journal
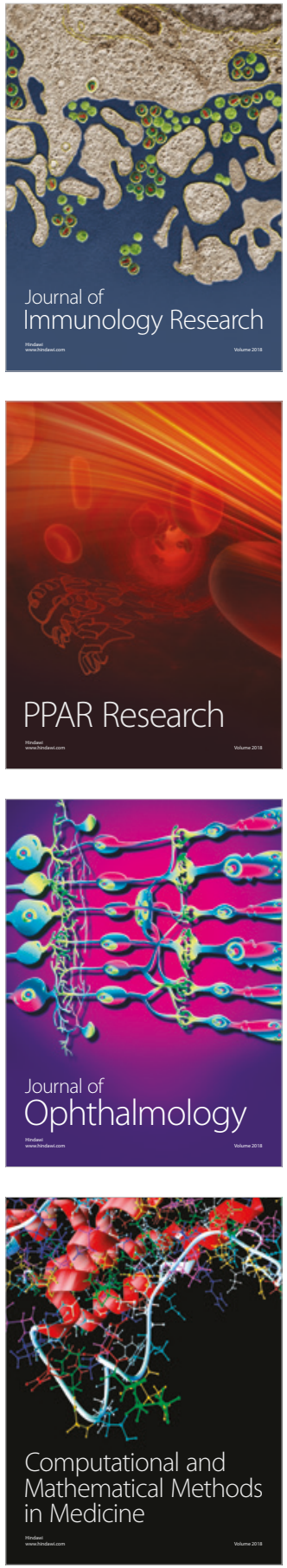

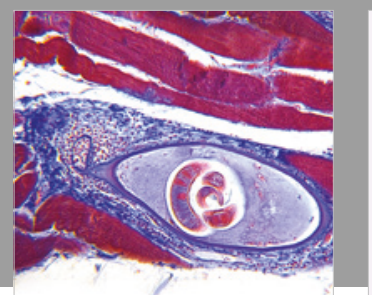

Gastroenterology Research and Practice

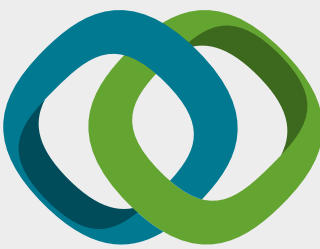

\section{Hindawi}

Submit your manuscripts at

www.hindawi.com
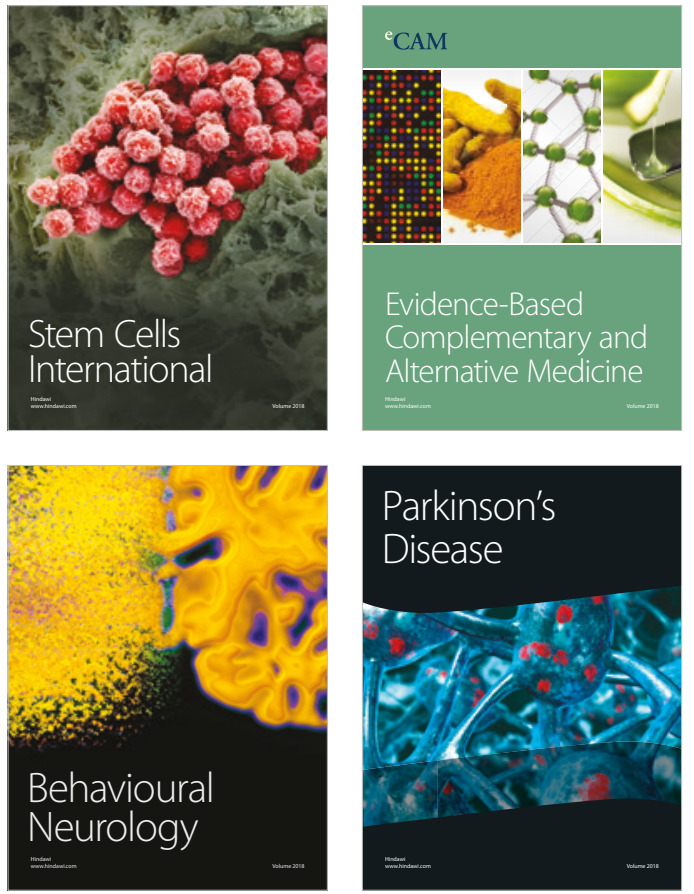

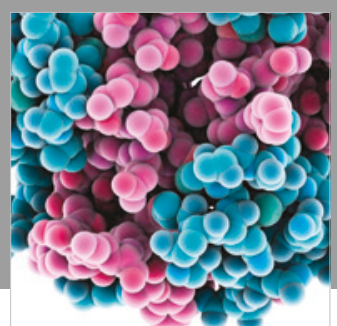

ournal of

Diabetes Research

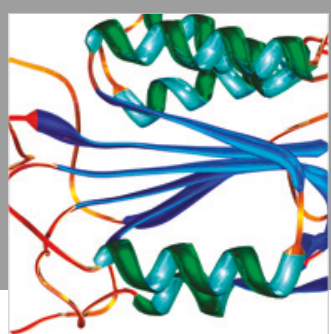

Disease Markers
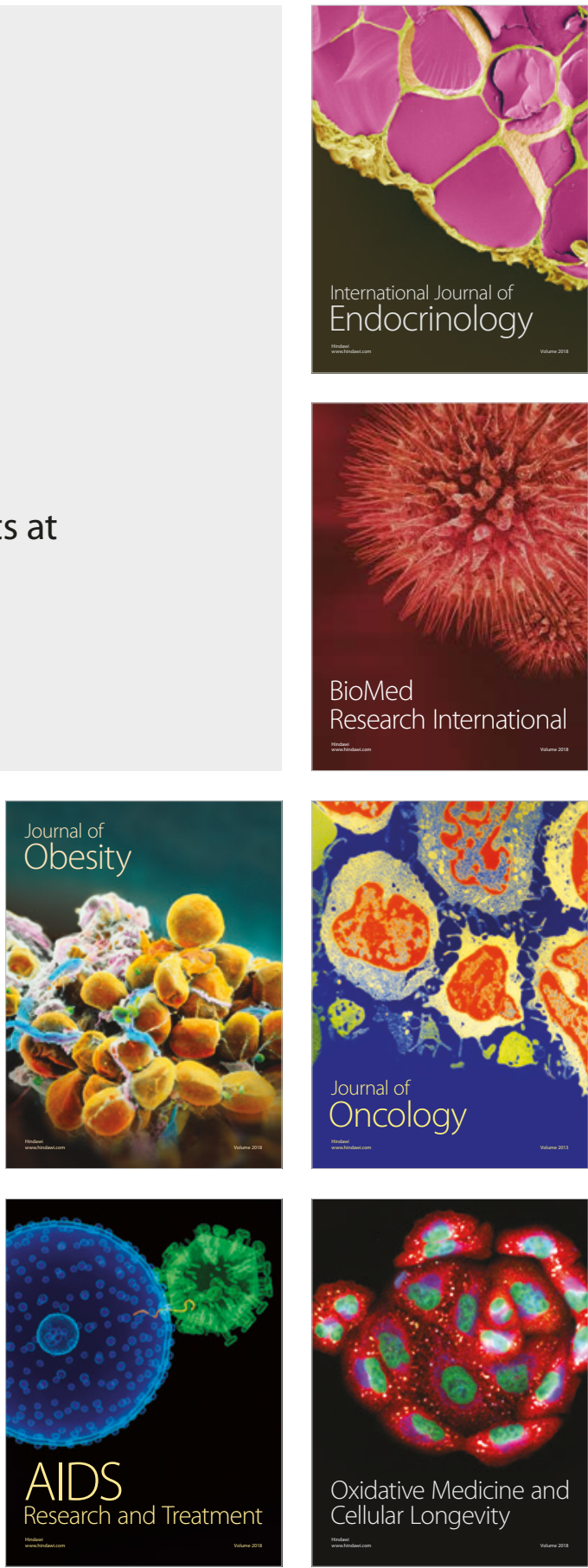